International Journal of Computer Science \& Information Technology (IJCSIT) Vol 3, No 6, Dec 2011

\title{
MOBILE GEOGRAPHIC INFORMATION SYSTEMS: A CASE STUDY ON MANSOURA UNIVERSITY, EGYPT
}

\author{
Asmaa Ahmed Hussein ${ }^{1}$, Elkhedr Hassan Eibrahim ${ }^{2}$, Aziza Asem ${ }^{1}$ \\ ${ }^{1}$ Faculty of Computer Sciences and information systems, Information Systems \\ Department, Mansoura University, Mansoura, Egypt. \\ AsmaaAhmed2010@hotmail.com, Aziza_Asem2@hotmail.com \\ ${ }^{2}$ Department of Geology and Geophysics, College of Science, King Saud University, \\ KSA \\ eibrahimeksu.edu.sa
}

\begin{abstract}
With the rapid development of GIS, embedded technology and wireless communication technology, mobile GIS becomes an active research area in the field of GIS. Information collection is one of the most promising application directions of mobile GIS. The purpose of this paper is to study the field of geographical information systems to propose a contribution in solving one of the bottlenecks that stumbles researchers from getting benefit of this field.

This research used WAP technology to facilitate the communication and data transfer between the end user and the GIS server. This enables the authors to solve the small storage area problem of mobile devices. The basic idea of the suggested solution is based on saving the layers and the database on the GIS server and executing queries on the Server instead of using the mobile storage that's so small comparing with PCS, so we saved the storage area in mobiles. The small storage area of mobiles is a great problem facing this research area.

Two prototypes were built on Mansoura University as a case study. The first one is an execution of location-based systems (LBS) and the second one is an execution of field-based systems (FBS). Applying the first prototype has proved to achieve its aims in helping the University's management in making surveys on all the distributed computers in the university while they are in their offices without the need to go to the site. Applying the second one has proved that the new comers found it's useful in helping them get the information they needed as well as telling them how to get there from here without any trouble.
\end{abstract}

KEYWORDS: Mobile GIS, location based services (LBS), field -based systems (FBS), Wireless Application Protocol (WAP).

\section{INTRODUCTION}

The convergence of the rapidly progressing fields of wireless communication and lightweight hardware coupled with technologies like geographic Information Systems (GIS) and global positioning systems (GPS) has led to the emergence of a new field called Mobile GIS [6]. Mobile GIS is bringing fundamental changes to the way geography is utilized and data is handled in mobile environments. It is extending the enterprise GIS by providing its users the ability to bring work with them, off the office environment . it is bridging the gap between working in the office and working on the move . users of mobile GIS can now retrieve,

DOI : $10.5121 /$ ijcsit.2011.3613 
manipulate and update enterprise data right from their PDA's anytime, from any where in the world. The resulting benefit is consistently improved productivity. Examples include real time access and tracking of shipments, emergency services, car navigation services, real time stock quotes, field services etc. With the proliferation of mobile GIS applications, the emphasis is now laid on spatial data; data that have the characteristic location attribute in addition to other attributes they may have[3].

The purpose of the paper is to propose a solution of mobile GIS for information collection by studying a detailed function of each component.

\section{DEFINITION OF MOBILE GIS}

The mobile GIS do not have a concrete definition, the same thing happened to GIS many years ago. There are many disciplines and technologies that can be related to mobile GIS, such as LBS (Location Based Services) [3]. The same issue is similar to mobile device, which has many characteristics that differ from one device to another.

Assuming that a mobile device is a portable device with mobile communication capabilities, processor, RAM, and local storage hard disk, can surf the internet and be connected to GPS device, Smartphone, PDA (Personal Data Assistant) and Pocket PC are samples of mobile devices[5].

Mobile GIS will be defined as the ability of mobile device to display geospatial data, and receive, process, and retrieve the GIS requests of mobile user. The most common framework for mobile GIS is to be considered as an extension to Web-GIS, where the GIS requests of the mobile user are processed via internet web browser.

Mobile GIS could be divided into two types according to the way of data access and information collection. Mobile GIS types are Field-based GIS and Location-based services.

Field-based GIS focuses on GIS data collection, and data validation and the updates in the field, such as adding new point data or changing the attribute tables of an existing GIS dataset, while Location-based services focus on business-oriented location management functions, such as navigation, street routing, finding a specific location, or tracking a vehicle[7].

\section{Field-BASEd GIS PRototype:-}

Most field-based GIS applications are used to edit or change the original GIS data or modify their attributes, so the prototype was designed to be used in collecting information from the field, modify the spatial database and attribute database as well. "Mansoura University" was chosen as a case study.

\subsection{DESIGN IDEA:-}

\subsubsection{SYSTEM ARCHITECTURE:}

Mobile GIS for field -based GIS and information collection is not an application of computing, but an integrated system. It is composed of mobile terminal device (Client) and service center (Server), joined through wireless communication networks and Internet between them, as shown in figure 1. There are some basic simple operations and requests that mobile terminal needn't ask the service centre and can achieve them independently. There're other operations that are complicated functions and can't be achieved by the mobile terminal which needs to be referred to the service center to deal with the request and returns the results. 


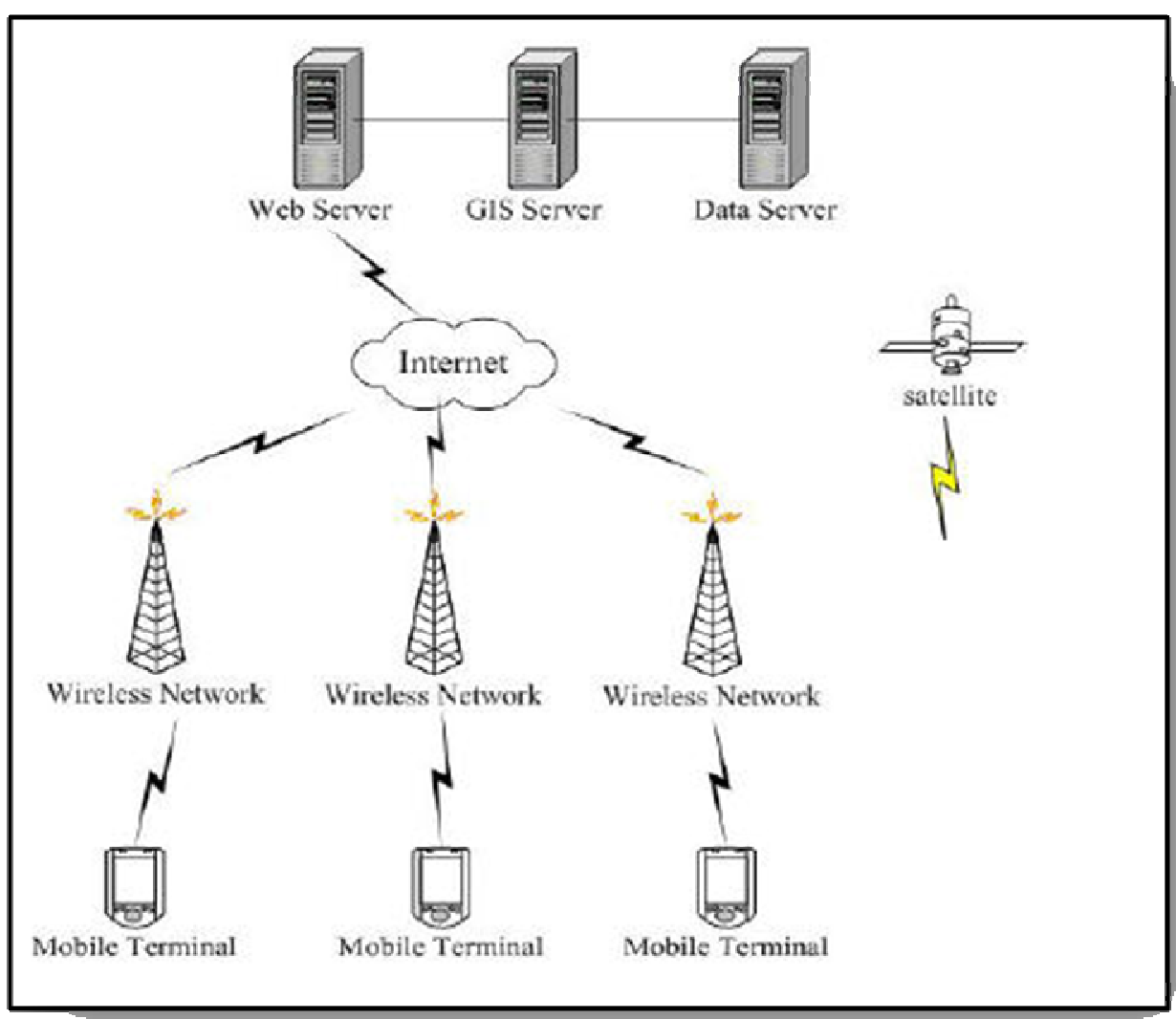

Figure 1. System architecture chart

The mobile terminal can transmit information, project maps, execute simple analysis, execute query and visualize information. While Server side (Service Centre) can update database, execute complicated analysis, transmit data and access the GIS from web or desktop system.

In the designed prototype for Mansoura University, the server side stores the Spatial database (shape files) and the attribute data (database tables).

The client side makes queries that are executed in the server and the results are transmitted back to the client side. We could distinguish between two types of users that act in the client side. The first type of users are researchers that use the application to survey the real world, collect data, publish data to the application and update the database(spatial or attribute). The other type of users is new comers that use the application to get information about the faculties and use the application to know how to get there from here. So we could summarize that in figure 2 that shows the proposed framework for field-based GIS. 
International Journal of Computer Science \& Information Technology (IJCSIT) Vol 3, No 6, Dec 2011

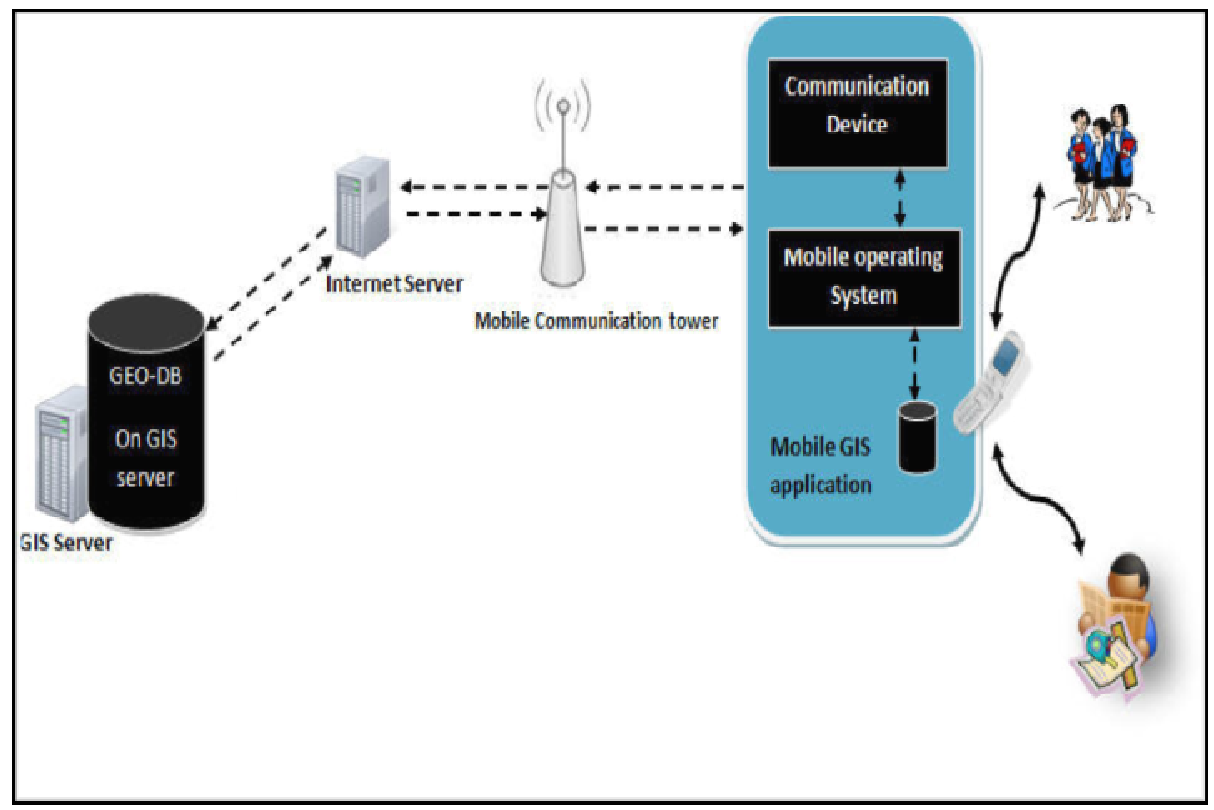

Figure 2. Proposed framework for field-based GIS.

\subsection{Spatial Data Management:-}

The core of building any GIS system is how to manage the spatial data. After analyzing the map of Mansoura University as the present case study, the map was divided into four thematic layers. The five layers are faculties, green_area, hospitals, roads and organizations. These layers manage different spatial data types; points, lines or polygons. As shown in Figure 3 , the desktop GIS show the four map layers.

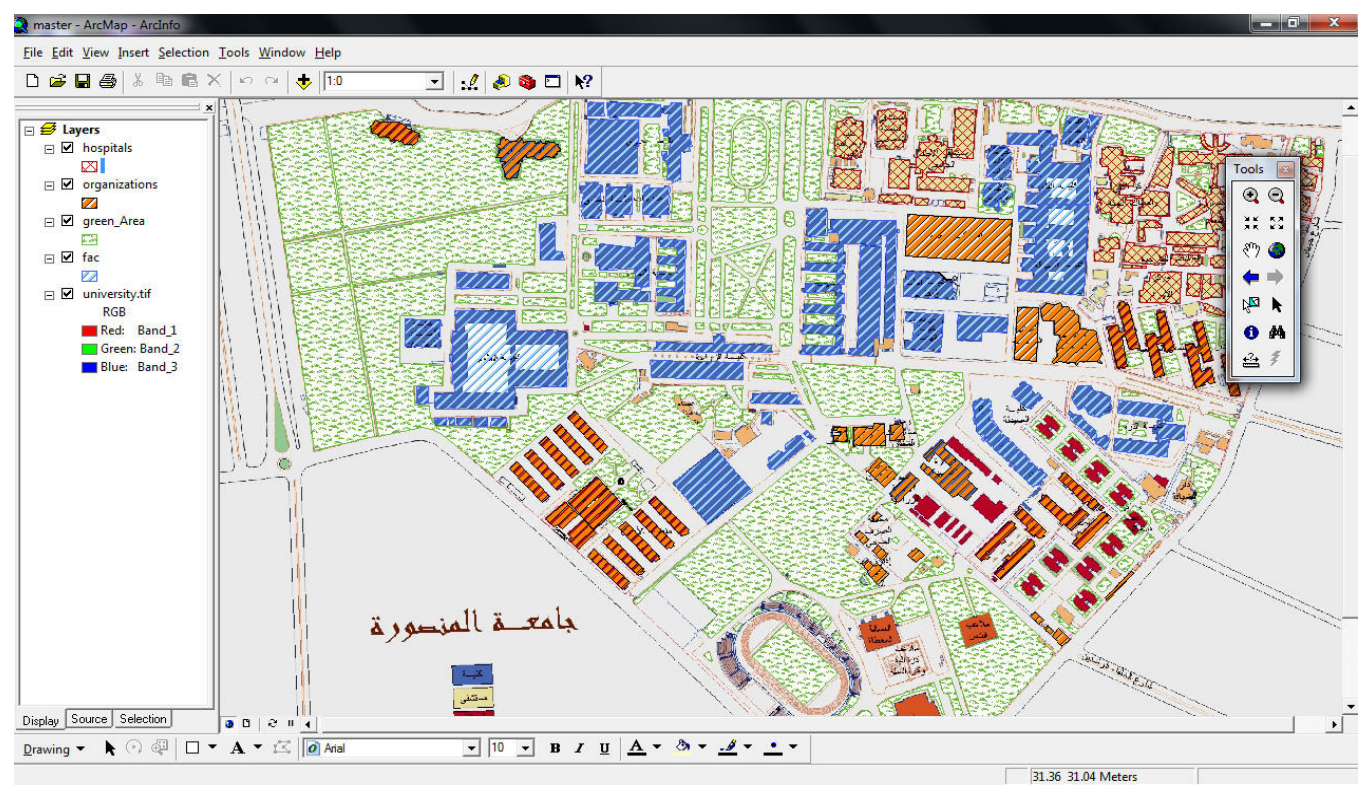

Figure 3. The map layers in Desktop GIS. 
International Journal of Computer Science \& Information Technology (IJCSIT) Vol 3, No 6, Dec 2011

To publish the desktop GIS system into a mobile GIS system, we used ArcGIS Server mobile SDK with .NET Framework and ArcPad. For example, if a new comer needs to know how to go from current place where helshe stays (e.g. desktop GIS stores x,y of the selected place) to the faculty of Information systems, mobile GIS will act as shown in Figure 4.

When the user places this query, the mobile device connects through the cellular network to access the GIS server where the map layers are stored and the GIS services are executed. Then the result will be sent back to the user .
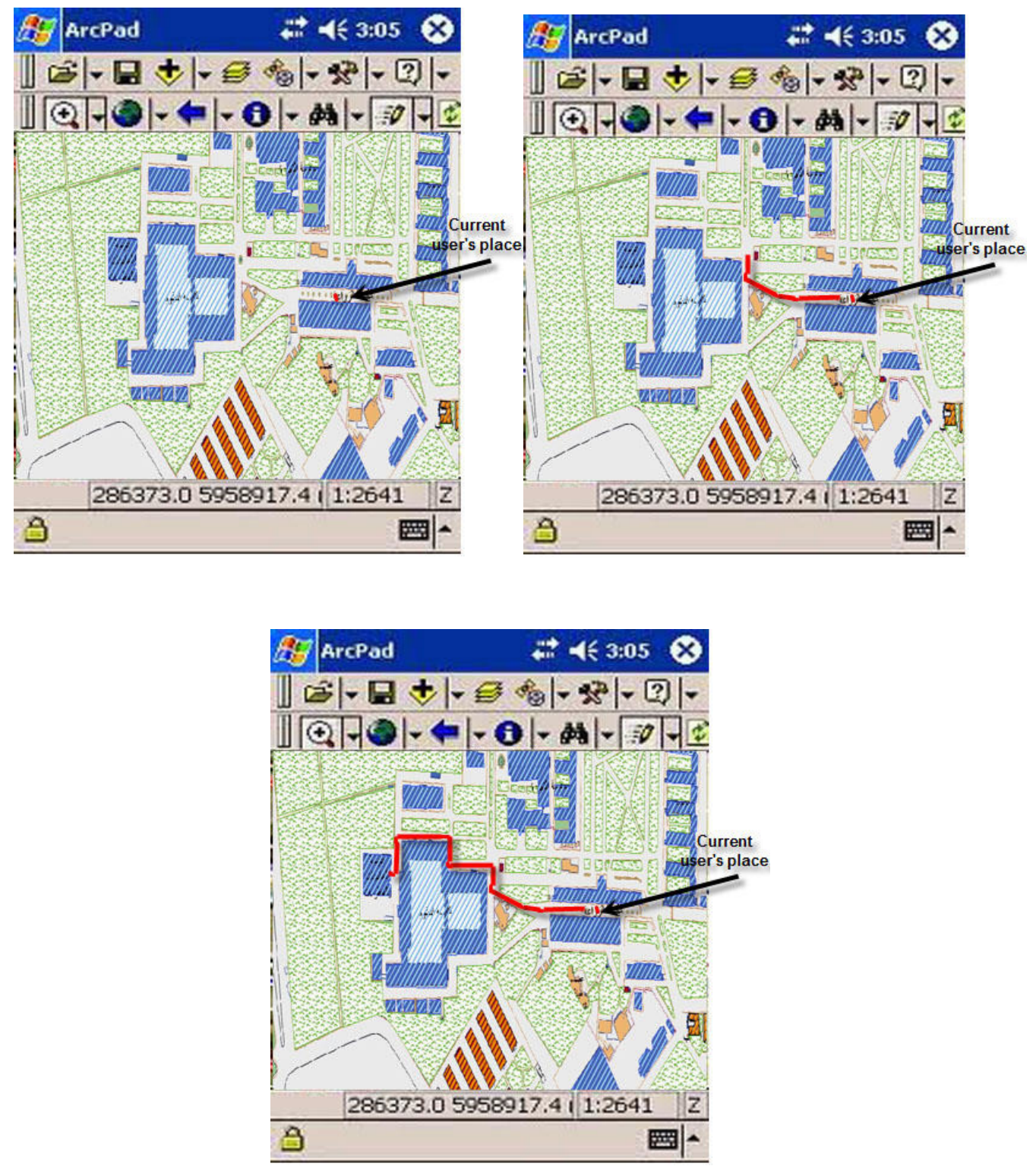

Figures 4. Routing in mobile GIs shown in ArcPad environment. 
International Journal of Computer Science \& Information Technology (IJCSIT) Vol 3, No 6, Dec 2011

\section{LOCATION-BASED GIS SYSTEMS:-}

Location Based Services (LBS) is a relatively new, growing technology field that focuses on providing information via mobile and field units based on individual spatial positions. Location-based services (LBS) rarely change original GIS datasets but rather use them as the background or reference maps for navigation or tracking purposes. LBS always uses on spatial positioning either a GPS or a cellular telephone to locate the user [1].

\subsection{SYSTEM ARCHITECTURE:}

LBS rely mainly on sensors. These sensors are instrumental in generating information that enables automatic extraction of information about the surrounding environment. In our case study, sensors should be connected to each PC in the university's network to detect any problem facing the users and to broadcast the problem to the specialists that are ready to fix it in the university's main center. Because of the complexities that face us in connecting such sensors, an alternative scenario was proposed for the information collection technique. This scenario simulates the existence of the sensors.

The proposed framework works as follows. The network map of the University with all the PCs connected to it was digitized firstly and the control points (TIC) are taken by GPS and were entered to the maps to give the real estimations. The attribute database were built and connected to the maps using ArcGIS Desktop. The desktop GIS application was published to Web-GIS using ArcGIS Server with .Net framework. The Web-GIS system was connected to SMS service provider to serve the SMS delivery.

\subsection{Spatial Data Management:-}

Two faculties of the university were taken as a sample to apply the following prototype. The faculty of Sciences and the faculty of information systems and computer sciences were chosen. We converted its floors, rooms and PCs to map layers. Then, they were connected to the data base. After that the desktop GIS was published to web-GIS with which the SMS will be sent. When there's a problem in any PC, the user will use any PC in the university's network to broadcast a problem. At this point, the alternative scenario will act on. When user selects a specific PC to place a problem, the web-GIS will connect to ArcGIS topology services to create the address of the PC and put it in the body of the SMS plus the problem that the user states. The addresses of the PCs are not stored in a database because of the need to reduce the database storage in web and mobile GIS. When a problem is stated, the PC stills alarming on the PC in the web-GIS till the administrator stops it. The administrator uses the WAP service on his mobile device to stop the alarm. When this is done a serial number of the mobile device is stored in a log file to save who stopped it. This scenario is shown in figure 5 . 

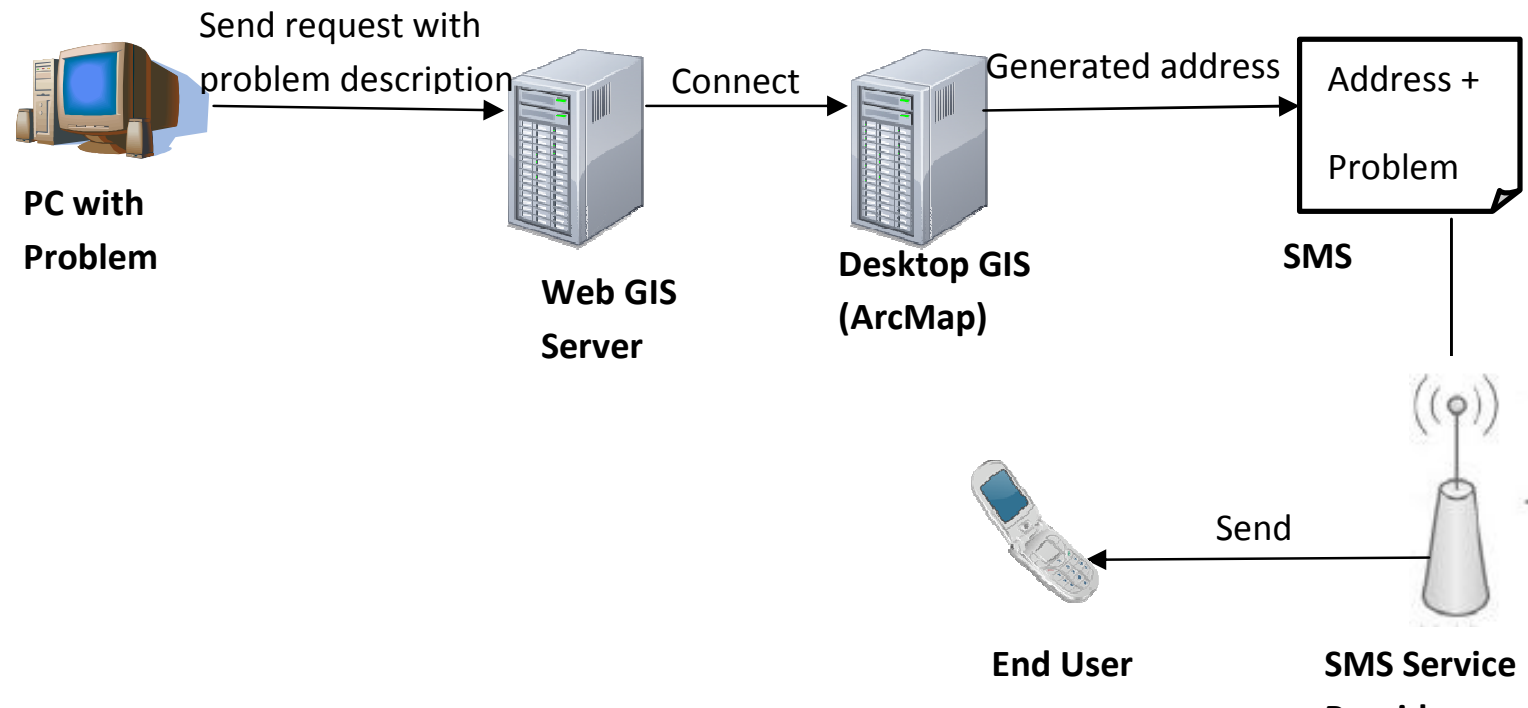

Figure 5. Proposed framework for location-Based system.

Provider

As shown in figure 6, when a computer is selected and be clicked in, a summary menu appears showing the computer ID, computer IP, the Support Name, and the created address . then a new problem could be added or the history of problems could be shown. If a new problem is added, it will be stored in a log file and a SMS will be sent immediately by connecting to the SMS service provider centre.

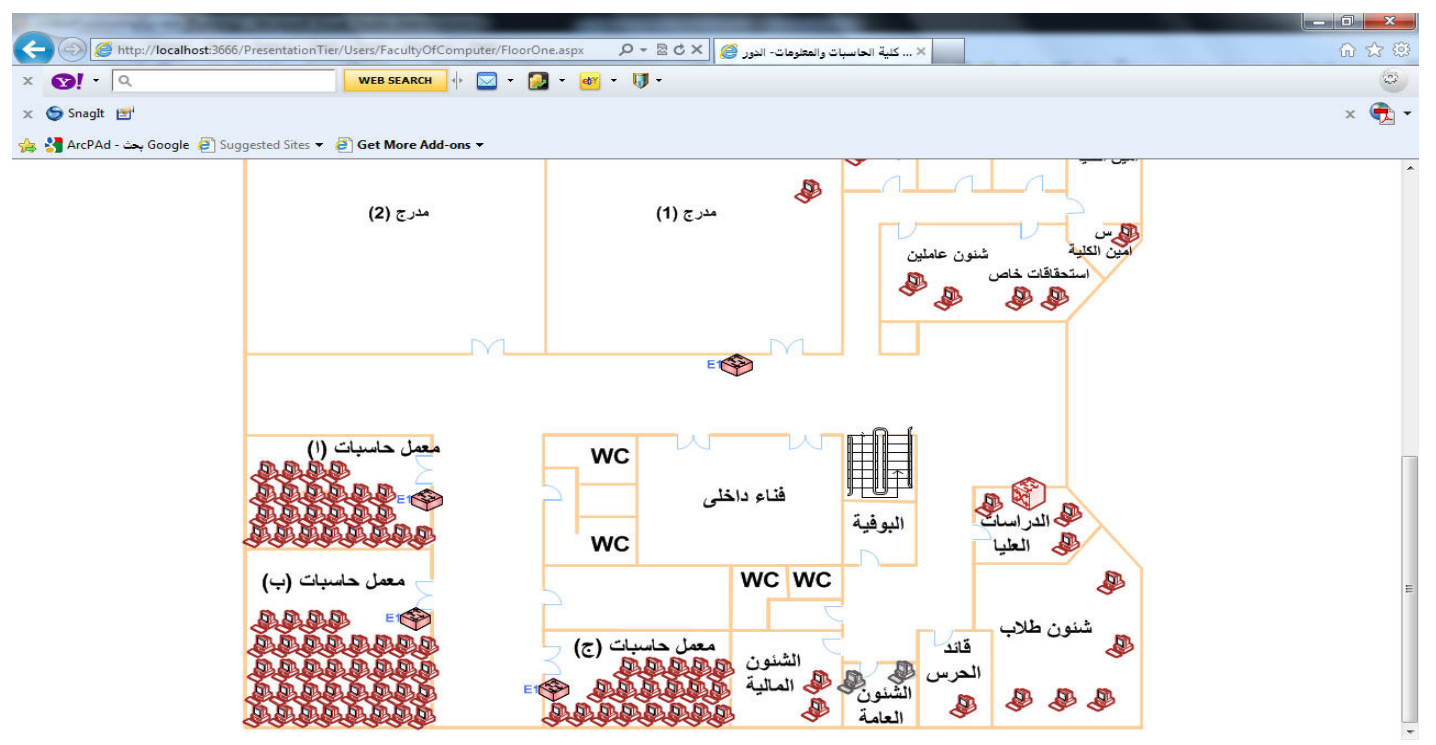


Summery

Computer ID 21

Computer IP 10.16 .20 .175

Support Name Administrator

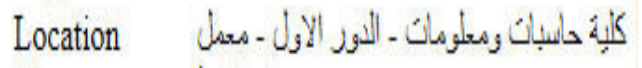

Name

\section{Action}

\section{Issue Add}

\section{Problem Description}

\section{View Report Add Problem}

Save And Send SMS

Figure 6. Screen Shots of LBS prototype.

\section{Conclusion:}

The main goal of the work described in this paper was to investigate the possibility of designing and implementing a mobile system, able to visualize and manipulate geographic information, with the interoperability of GIS systems in mind, i.e., by using formats and open standards. To assess these premises, architecture was designed and a two prototype called Mansoura University M-GIS was implemented.

The results of the M-GIS assessment conducted allow us to conclude that it is possible, but with some limitations. The main limitations are related with the amount of information that can be processed by the mobile client. The first limitation of the current devices have to do with the amount of memory needed to visualize a given map and the second with the screen resolution needed to visualize the maps well.

These limitations clearly indicate that M-GIS success is attached by the way with the used mobile device capability of the client (i.e. the amount of memory, the screen resolution, the network coverage, etc). On the other hand, the test of the first prototype gave an excellent feedback from student on whom we have tested it. Also the seond prototype was tested on employees in the University management whom found that it could give them a new chance to bring their work off offices, so they could improve their achievements.

\section{REFERENCES:}

[1] D. M. Krum, W. Ribarsky, C. D. Shaw, L. F. Hodges, and N. Faust (2001), "Situational Visualization, Virtual Reality Software and Technology", Banff, Canada, pp. 143-150, ACM.

[2] E.Y.Chen and T. M. Chan, "A Space-Efficient, Algorithm for Segment Intersection", Proc.15th Canadian Conference on Computational Geometry, 2003, pp. 68-71. 
International Journal of Computer Science \& Information Technology (IJCSIT) Vol 3, No 6, Dec 2011

[3] Jekkin.D.Shah, (2009) "Semantic Query Cashing in Mobile Environments", computer Science and electrical engineering journal, Vol. 12, pp. 12.

[4] Li L, Li C, Lin Z (2002): "Investigation on the Concept Model of Mobile GIS". Symposium on Geospatial Theory, Processing and Applications, Ottawa.

[5] Mountain, D.M., J.L.Y. Tsui, and J.F. Raper. Modelling accessibility via transportation networks based upon previous experience: a Geographically Weighted Regression approach. in Geocomputation 2007. in press. National University of Ireland, Maynooth, Ireland: National University of Ireland.

[6] Scholtz, J., Metrics for evaluating human information interaction systems. Interacting with Computers, 2006. 18(4): p. 507-527.

[7] S.Shekhar, Y. Huang, and J. Djugash. "Design Algorithms for Vector Map Compression", In Proceedings of Data Compression Conference, 2002 